\title{
Evaluating quality and completeness of gastrectomy for gastric cancer: review of surgical videos from the public domain
}

\author{
Matthew Dixon ${ }^{1}$, Vanessa Palter ${ }^{2}$, Savtaj Brar ${ }^{1,3}$, Natalie Coburn ${ }^{1,4,5}$ \\ ${ }^{1}$ Department of Surgery, University of Toronto, Toronto, ON, Canada; ${ }^{2}$ St. Michaels Hospital, Toronto, ON, Canada; ${ }^{3}$ Mount Sinai Hospital, \\ Toronto, ON, Canada; ${ }^{4}$ Sunnybrook Health Sciences Centre, Toronto, ON, Canada; ${ }^{5}$ Sunnybrook Research Institute, Toronto, ON, Canada \\ Contributions: (I) Conception and design: All authors; (II) Administrative support: M Dixon; (III) Provision of study materials or patients: All authors; \\ (IV) Collection and assembly of data: M Dixon, V Palter, S Brar; (V) Data analysis and interpretation: All authors; (VI) Manuscript writing: All \\ authors; (VII) Final approval of manuscript: All authors. \\ Correspondence to: Dr. Natalie Coburn. Sunnybrook Health Sciences Centre, T2-11, 2075 Bayview Avenue, Toronto, ON M4N 3M5, Canada. \\ Email: Natalie.Coburn@sunnybrook.ca.
}

\begin{abstract}
Background: YouTube ${ }^{\circledR}$ has provided a forum to share surgical videos in the public domain which may be used for education. The quality of surgery and accompanying educational material is unknown. YouTube ${ }^{\circledR}$ videos of gastrectomy with D2 lymphadenectomy (D2-LND) for gastric cancer were evaluated for quality and completeness.

Methods: YouTube ${ }^{\circledR}$ was searched using the terms “D2 lymphadenectomy" and "Gastric Cancer" for open and laparoscopic videos. The Korean Laparoscopic Gastrointestinal Surgical Society (KLASS) outlined 22 steps that define quality and completeness of D2-LND. These guidelines were used to score D2-LND for each video. Four physician reviewers independently scored each surgical video. Scores were compared using Student's $t$-test.
\end{abstract}

Results: Ten laparoscopic and 10 open surgery videos were assessed. Each video was scored for quality and completeness and assigned a score out of 22. Mean score for open D2-LND was 15 (95\% CI: 12.54-17.46). Mean score for laparoscopic D2-LND was 15.4 (95\% CI: 14.34-16.46; P=0.77). The most consistently performed steps were the dissection of lymph node stations 1, 3, 4 and 5. The most commonly omitted steps were the dissection of lymph node station 6: exposure and identification of the lowest anterior superior pancreaticoduodenal vein; removal of the prepancreatic soft tissues above the lowest anterior superior pancreaticoduodenal vein; removal of the prepancreatic soft tissues above the level of the bifurcation of the anterior superior pancreaticoduodenal vein and right gastroepiploic vein.

Conclusions: There is a wide range of quality and completeness of D2-LND videos. On average, D2LND videos are only two-thirds complete.

Keywords: YouTube; D2; lymphadenectomy; gastrectomy

Received: 05 November 2019; Accepted: 02 January 2020; Published: 25 October 2021.

doi: $10.21037 / \operatorname{tgh} .2020 .01 .01$

View this article at: http://dx.doi.org/10.21037/tgh.2020.01.01

\section{Introduction}

Worldwide, gastric cancer is a leading cause of mortality (1), with surgery representing the only potentially curative treatment. Despite 5-year survival rates of $40-60 \%$ in series from Asia and Europe $(2,3)$. Five-year survival in North America is much worse, approximately $31 \%$ (4). Reasons for these survival differences are unclear, but may be related to low-volume surgeons performing resections, less aggressive surgery, poor staging, and differences in biology (5).

In addition to resection of the tumor, extent of lymphadenectomy may affect survival. Non-randomized series from Asia and Europe have reported improved survival associated with the more extensive D2 lymphadenectomy (6-10) compared to the more limited D1 lymphadenectomy, 
which is commonly performed in North America. On the other hand, multiple randomized controlled trials have failed to show a survival benefit from the D2 lymphadenectomy, with increased morbidity and mortality over the D1 lymphadenectomy. Much of this morbidity has been attributed to performing a distal pancreatectomy and splenectomy as part of the D2 lymphadenectomy, and that this may erode any potential survival benefit from the more extended lymphadenectomy. Songun et al. have published 15-year follow-up data for the Dutch trial of D1 vs. D2 lymphadenectomy, and on subgroup analysis, patients who avoided pancreatosplenectomy and underwent D2 lymphadenectomy experienced a 15 -year survival of $35 \%$, compared to $22 \%$ for patients who underwent a D1 lymphadenectomy $(\mathrm{P}=0.006)$ (11).

As the D2 lymphadenectomy is a seldom-performed procedure in most Western centers (12), many new trainees and a new generation of surgeons performing gastrectomy for gastric cancer have little experience with it. This opens the opportunity for other educational tools to help this new generation of surgeons gain some knowledge regarding the D2 lymphadenectomy. Surgical videos available on the public domain through sites such as YouTube pose an obvious and potentially useful means of gaining exposure to this procedure and increasing familiarity with it.

However, there are potential problems with using surgical videos from the public domain to fill knowledge and experience gaps. Many videos on YouTube seem to be based on personal experience, and with a lack of a peer-review process, there is the potential for posting of inaccurate or misleading health information. To date, there has been no study that has evaluated the quality and completeness of D2 lymphadenectomy in surgical videos using an established guideline as a benchmark. Therefore, our study objectives were to review publically available surgical videos of gastrectomy for gastric cancer, and to score these videos using previously established scoring systems for quality and completeness of D2 lymphadenectomy. We also sought to compare differences between videos for open and laparoscopic D2 lymphadenectomy. Our hypothesis was that there will be a wide range in quality and completeness of D2 lymphadenectomy depicted in both laparoscopic and open videos.

\section{Methods}

The website YouTube ${ }^{\circledR}$ is a publically available website for video blogs. YouTube ${ }^{\circledast}$ was searched for videos depicting a subtotal or total gastrectomy with D2 lymphadenectomy. YouTube ${ }^{\circledR}$ was searched on October 20, 2016 for the search terms "D2 lymphadenectomy" and "Gastric Cancer." Twenty videos were selected for review: 10 videos depicting open resection, and 10 videos depicting laparoscopic resection. Criteria for selection were (I) videos no older than 2010, and (II) videos with a minimum of 250 views. These data are available directly from the YouTube ${ }^{\circledR}$ website.

The Korean Laparoscopic Gastrointestinal Surgical Society (KLASS) have established guidelines to ensure the quality of D2 lymphadenectomy in their ongoing KLASS trials examining the efficacy of laparoscopic D2 lymphadenectomy in locally advanced gastric cancer patients compared with the open procedure (13). These guidelines contain 22 distinct steps that are a marker for quality and completeness of D2 lymphadenectomy (Table 1). These guidelines were used to evaluate all surgical videos according to whether a specific step was performed during the D2 lymphadenectomy depicted in each video, and therefore rating the quality and completeness of the D2 lymphadenectomy in each video. Four physician reviewers independently scored each surgical video according to the KLASS-02 guidelines to quantify quality and completeness of each video. All videos were reviewed a minimum of 3 times by 3 different physicians. Mean scores were calculated for each video. Scores for open and laparoscopic surgery were compared using Student's $t$-test.

Research Ethics Board (REB) approval was obtained through Sunnybrook Health Sciences Centre.

\section{Results}

A total of 1,110 videos were identified using the search terms "gastric cancer" and "D2 lymphadenectomy." Based on the search criteria described previously, 10 open and 10 laparoscopic videos were selected. Each video was observed for quality and completeness based on performance of 22 distinct steps and assigned a score out of 22 .

The mean scores for quality and completeness of D2 lymphadenectomy were calculated for all open and laparoscopic videos out of a total score of 22 , based on the 22 distinct steps outlined in Table 1. The mean score for open D2-lymphadenectomy was 15 out of 22 (95\% CI: 12.54-17.46), while the mean score for laparoscopic D2lymphadenectomy was 15.4 out of 22 (95\% CI: 14.34 16.46) $(\mathrm{P}=0.77)$. Only one video $(5 \%)$ was successful in achieving a score of 22 .

Results for each specific maneuver, as outlined by the 
Table 1 Evaluation criteria for completeness of D2 lymphadenectomy according to KLASS-2 trial

Procedure
1. Total omentectomy
2. Division of left gastroepiploic artery
(it is not necessary to dissect the root of
left gastroepiploic artery if the tumor is
located in lower third of the stomach)

3. Appropriate extent of No. 6 lymph node (LN) dissection

4. Appropriate extent of No. 5 LN dissection

\author{
5. Appropriate extent of No. 12a LN \\ dissection
}

\section{Appropriate extent of No. 8a LN dissection}

7. Appropriate extent of No. $9 \mathrm{LN}$ dissection (resection of the celiac plexus is not necessary)

8. Appropriate extent of No. 7 LN dissection

\section{Appropriate extent of No. 11p LN dissection}

10. Prevention of pancreatic injury during suprapancreatic LN dissection

11.Appropriate extent of No. 1 and $3 \mathrm{LN}$ dissection

\section{Station Requirement}

No injury was made to the any other organ

4Sb The left gastroepiploic artery and left gastroepiploic vein are divided at least below the bifurcation of the first gastric branch

No injury was made to the colon of splenic flexure

4d The branch of right gastroepiploic artery and vein are retrieved

6 The right gastroepiploic vein is divided just above the bifurcation of the anterior superior pancreaticoduodenal vein and the right gastroepiploic vein

The right gastroepiploic artery is divided just peripheral to the bifurcation of the right gastroepiploic artery and the anterior superior pancreaticoduodenal artery

The lowest anterior superior pancreaticoduodenal vein is identified and exposed

The prepancreatic soft tissues above the lowest anterior superior pancreaticoduodenal vein are completely removed

The prepancreatic soft tissues above the level of the bifurcation of the anterior superior pancreaticoduodenal vein and right gastroepiploic vein are completely removed

No injury was made to the pancreatic parenchyma

5 The root of right gastric artery is identified and exposed

12a The lower half of the proper hepatic artery is exposed; at least its anterior and left surfaces

The left side of the portal vein is identified and exposed and soft tissues are completely removed

8a The common hepatic artery is exposed; at least its anterior and superior surfaces

The soft tissues above the upper edge of the pancreas are completely removed

9 The retroperitoneal membrane is divided along the boundary between the right crus and the soft tissues around the celiac trunk to completely dissect No. 9 LNs

7 The root of the left gastric artery is exposed and ligated

$11 \mathrm{p}$ The proximal half of the splenic artery is exposed, from its root to the site where the meandering splenic artery is in the closest vicinity to the stomach

The splenic vein is identified and exposed, or at least the dorsal side of pancreatic parenchyma is exposed

No pancreatic injury by heat of energy devices and/or assistant's forceps was caused

1, 3 The soft tissue attached to the lesser curvature side of gastric wall is completely removed

No esophageal and/or gastric injury by heat of energy devices and/or blind manipulation was caused 
KLASS-2 trial, are reported in Table 2. These data are reported as the proportion of videos in which each individual step is scored as having been completed. Generally, the proportion of videos where the step was documented as completed was consistent between laparoscopic and open videos.

The most consistently performed steps were the dissection of lymph node stations 1, 3, 4 and 5. The only statistically significant difference between videos was in performance of a total omentectomy, which was more commonly performed in open videos $(\mathrm{P}=0.012)$. The steps in which both laparoscopic and open videos lacked completion centered mainly on proper dissection of lymph node station 6. Specifically, most videos failed to demonstrate adequate exposure of the lowest anteriorsuperior pancreaticoduodenal vein, removal of the prepancreatic soft tissues above the lowest anterior-superior pancreaticoduodenal vein, and proper demonstration of the bifurcation of the anterior-superior pancreaticoduodenal vein and the right gastroepiploic vein with removal of the prepancreatic soft tissue in this area.

As per the Japanese Gastric Cancer Associations guidelines (14), dissection of lymph node station 1 is standard for subtotal gastrectomy. The KLASS guidelines combine dissection of station 1 and station 3 together. We performed a subgroup analysis of this station for videos depicting subtotal and total gastrectomy. In the open group, 2 videos depicted subtotal gastrectomy, while 8 videos depicted total gastrectomy. Mean scores for complete dissection of station 1 and 3 for subtotal gastrectomy was 0.67 , and 0.83 in the total gastrectomy $(\mathrm{P}=0.425)$. In the laparoscopic group, 3 videos depicted subtotal gastrectomy, while 7 videos depicted total gastrectomy. Mean scores for complete dissection of station 1 and 3 in the subtotal gastrectomy was 0.89 and 0.95 in the total gastrectomy $(\mathrm{P}=0.545)$.

\section{Discussion}

This study sought to quantify the quality and completeness of D2 lymphadenectomy depicted in laparoscopic and open videos available in the public domain. Quantification of quality and completeness was done using previously established standards employed in an ongoing clinical trial. As expected, a wide range of quality and completeness was discovered amongst the most viewed videos accessible from the public domain and were $68 \%$ complete for open D2 lymphadenectomies, and $70 \%$ complete for laparoscopic
D2 lymphadenectomies.

The only statistically significant difference between laparoscopic and open videos was observed in the rate of omentectomy, with this more commonly performed during open gastrectomy. One potential explanation for this is that during laparoscopic gastrectomy, with patients positioned in a reverse trendelenburg position, it is less cumbersome to leave the omentum attached to the transverse colon, and to enter the lesser sac adjacent to the gastroepiploic arcade. According to the Japanese Gastric Cancer Association guidelines, removal of the greater omentum is usually integrated in the standard gastrectomy for T3 or deeper tumors, while for T1/T2 tumors, the omentum that is greater than $3 \mathrm{~cm}$ away from the gastroepiploic arcade may be preserved (14). On the other hand, there is disagreement on the appropriateness of omentectomy during all gastrectomy (15), as the oncologic benefit of performing it is not well known. Therefore, the reason for this difference is likely technical in nature.

In both the open and laparoscopic videos, dissection of station 6, the infrapyloric lymph node station, was often incomplete. In particular, dissection of the lowest anteriorsuperior pancreaticoduodenal vein, removal of adjacent soft tissue and demonstration of this vein bifurcation with the right gastroepiploic vein was seldom demonstrated. The most likely explanation for the lack of completion of this step, according to the KLASS-02 criteria (13), is that surgeons in the videos depicted were careful to avoid injury to the pancreas and therefore, did not pursue dissection of the anterior-superior pancreaticoduodenal veins in this detail. The Japanese Gastric Cancer Association guidelines do not address dissection of station 6 in this detail (14), and the oncologic benefit of dissecting these vessels down to the pancreas is unknown.

The Internet has revolutionized public access to information on health and disease. It has also created opportunity for the medical community to disseminate information for the purposes of education. Specifically, public websites such as YouTube ${ }^{\circledR}$, have provided a forum to share surgical videos in the public domain. Making surgical videos available on the public domain can be used for education, not only for patients, but for health care practitioners as well. For example, use of surgical videos and remote connection through the internet, in low-resource settings, where presence of experienced faculty is limited and access to visiting faculty is sporadic, is feasible, effective, and well-accepted by both learner and teacher (16).

On the other hand, the quality of these videos can be 
Table 2 Results for scoring each dissection maneuver for a D2 lymphadenectomy based on the guidelines published by the Korean Laparoscopic Gastrointestinal Surgical Society (KLASS)

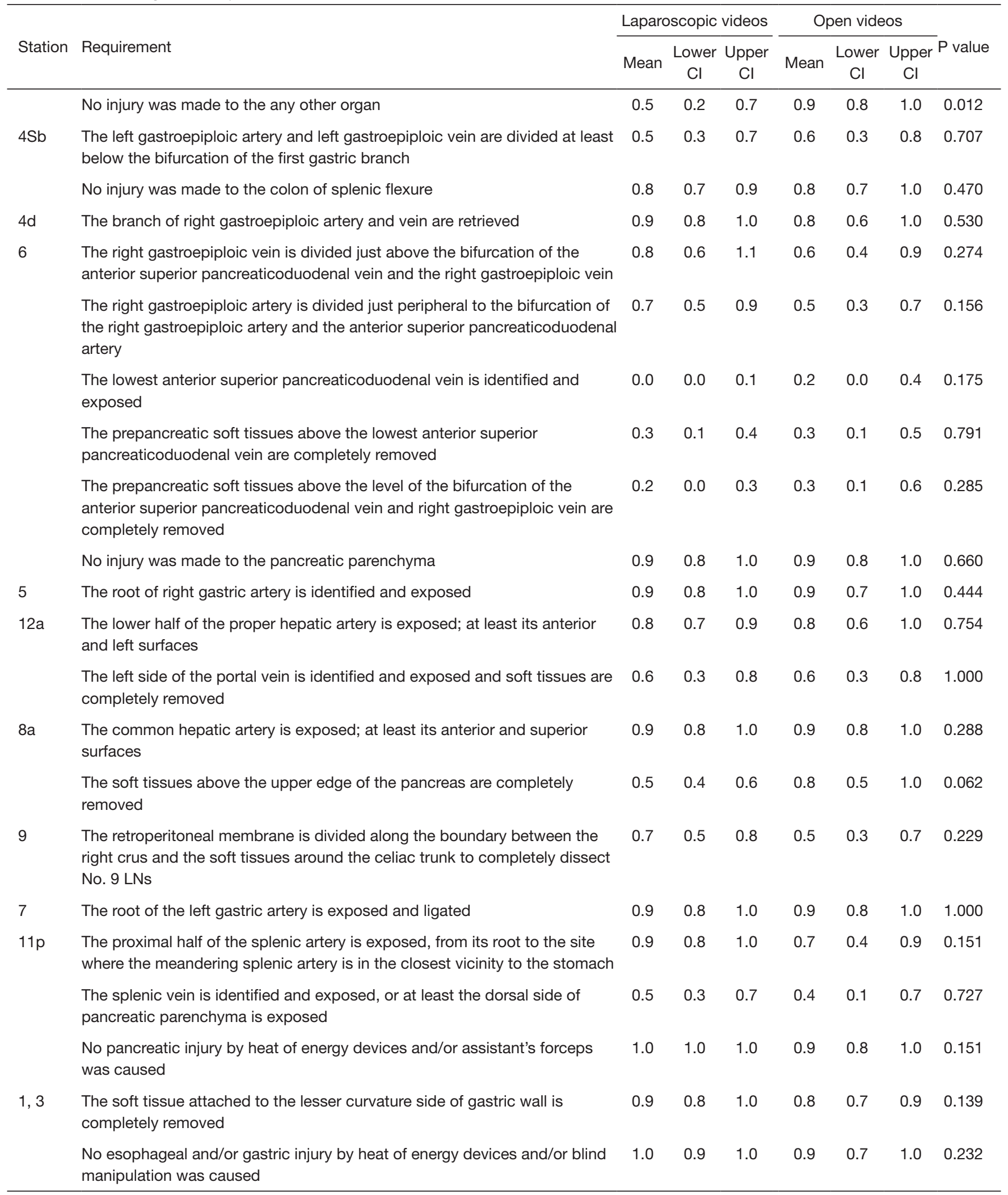


difficult to enforce when they are uploaded to the public domain. Many videos on YouTube ${ }^{\circledR}$ are available from international experts, but some of these videos are based on personal experience. The diversity of authorship and lack of a peer-review process on YouTube ${ }^{\circledR}$ has led to the posting of inaccurate or misleading health information (17-19). Strychowsky et al. (17) evaluated YouTube ${ }^{\circledR}$ as a patient source of information for pediatric tonsillectomy. They found a large number of videos on pediatric tonsillectomy on YouTube ${ }^{\circledR}$ with a variety of content ranging from very useful to not useful, and misleading. Complicated and uncommonly performed procedures, such as the D2 lymphadenectomy for gastric cancer, may be especially vulnerable to the posting of inaccurate or misleading information. Our study is the first to look at quality and completeness of videos depicting a D2 lymphadenectomy.

This study does have limitations. We used a limited sample with which to evaluate surgical videos. While other videos not viewed may in fact depict a complete D2 lymphadenectomy based on the KLASS-02 guidelines (13), we sought to evaluate relative impact by identifying the most commonly viewed videos as these are what many viewers may be using to judge what depicts a complete D2 lymphadenectomy. It was for this reason that we also did not search specialized websites such as WebSurg ${ }^{\circledR}$, or various society websites. It is our expectation that these sites may in fact contain videos depicting more complete D2 lymphadenectomies, because of an established peer review process. As such, we chose to focus on videos available in the public domain on YouTube ${ }^{\circledR}$ as these are more widely accessible by the general public and may represent a more utilized resource.

\section{Conclusions}

In summary, this is the first study that has sought to quantify the quality and completeness of D2 lymphadenectomy of videos available on YouTube ${ }^{\circledast}$. There is a wide range of quality and completeness of open and laparoscopic D2 lymphadenectomy videos. This reflects inadequate education on the quality and completeness of D2 lymphadenectomy. Improvements in surgical education, as well as online resources, may improve the quality and completeness of the D2-lymphadenectomy.

\section{Acknowledgments}

This article was presented in abstract format at the 12 th
International Gastric Cancer Congress 2017, Beijing, China, April 2017.

Funding: None.

\section{Footnote}

Conflicts of Interest: All authors have completed the ICMJE uniform disclosure form (available at http://dx.doi. org/10.21037/tgh.2020.01.01). The authors have no conflicts of interest to declare.

Ethical Statement: The authors are accountable for all aspects of the work in ensuring that questions related to the accuracy or integrity of any part of the work are appropriately investigated and resolved. Research Ethics Board (REB) approval was obtained through Sunnybrook Health Sciences Centre.

Open Access Statement: This is an Open Access article distributed in accordance with the Creative Commons Attribution-NonCommercial-NoDerivs 4.0 International License (CC BY-NC-ND 4.0), which permits the noncommercial replication and distribution of the article with the strict proviso that no changes or edits are made and the original work is properly cited (including links to both the formal publication through the relevant DOI and the license). See: https://creativecommons.org/licenses/by-nc-nd/4.0/.

\section{References}

1. Siegel RL, Miller KD, Jemal A. Cancer Statistics, 2018. CA Cancer J Clin 2018;68:7-30.

2. Kim JP. Surgical results in gastric cancer. Semin Surg Oncol 1999;17:132-8.

3. McCulloch P, Nita M, Kazi H, et al. Extended versus limited lymph nodes dissection technique for adenocarcinoma of the stomach. Cochrane Database Syst Rev 2004;18:CD001964.

4. Howlader N, Noone AM, Krapcho M, et al. SEER Cancer Statistics Review, 1975-2013. Bethesda, MD: National Cancer Institute, 2016.

5. Sasako M, Saka M, Fukagawa T, et al. Surgical treatment of advanced gastric cancer: Japanese perspective. Dig Surg 2007;24:101-7.

6. Degiuli M, Sasako M, Calgaro M, et al. Morbidity and mortality after D1 and D2 gastrectomy for cancer: interim analysis of the Italian Gastric Cancer Study Group (IGCSG) randomised surgical trial. Eur J Surg Oncol 
2004;30:303-8.

7. Hartgrink HH, van de Velde CJ. Status of extended lymph node dissection: locoregional control is the only way to survive gastric cancer. J Surg Oncol 2005;90:153-65.

8. Maruyama K, Okabayashi K, Kinoshita T. Progress in gastric cancer surgery in Japan and its limits of radicality. World J Surg 1987;11:418-25.

9. Mishima Y, Hirayama R. The role of lymph node surgery in gastric cancer. World J Surg 1987;11:406-11.

10. Pacelli F, Doglietto G, Bellantone R, et al. Extensive versus limited lymph node dissection for gastric cancer: a comparative study of 320 patients. Br J Surg 1993;80:1153-6.

11. Songun I, Putter H, Kranenbarg EM, et al. Surgical treatment of gastric cancer: 15-year follow-up results of the randomized nationwide Dutch D1D2 trial. Lancet Oncol 2010;11:439-49.

12. Macdonald JS, Smalley SR, Benedetti J, et al. Chemoradiotherapy after surgery compared with surgery alone for adenocarcinoma of the stomach or gastroesophageal junction. N Engl J Med 2001;345:725-30.

13. Hur H, Lee HY, Lee HJ, et al. Efficacy of laparoscopic

doi: $10.21037 / \operatorname{tgh} .2020 .01 .01$

Cite this article as: Dixon M, Palter V, Brar S, Coburn N. Evaluating quality and completeness of gastrectomy for gastric cancer: review of surgical videos from the public domain. Transl Gastroenterol Hepatol 2021;6:57. subtotal gastrectomy with D2 lymphadenectomy for locally advanced gastric cancer: the protocol of the KLASS-02 multicenter randomized controlled clinical trial. BMC Cancer 2015;15:355.

14. Japanese Gastric Cancer Association. Japanese gastric cancer treatment guidelines 2014 (ver. 4). Gastric Cancer 2017;20:1-19.

15. Brar S, Law $\mathrm{C}, \mathrm{McLeod} \mathrm{R}$ et al. Defining surgical quality in gastric cancer: a RAND/UCLA appropriateness study. J Am Coll Surg 2013;217:347-51.

16. Autry AM, Knight S, Lester F, et al. Teaching surgical skills using video internet communication in a resourcelimited setting. Obstet Gynecol 2013;122:127-31.

17. Strychowsky JE, Nayan S, Farrokhyar F, et al. YouTube: a good source of information on pediatric tonsillectomy: Int J Pediatr Otorhinolaryngol 2013;77:972-5

18. Pandey A, Patni N, Sing M, et al. YouTube as a source of information on the H1N1 influenza pandemic, Am J Prev Med 2010;38:e1-3.

19. Sood A, Sarangi S, Pandey A, et al. YouTube as a source of information on kidney stone disease. Urology 2011;77:558-62. 